\title{
Resolving the Nuclear Obscuring Disk in the Compton-thick Seyfert Galaxy NGC 5643 with ALMA
}

\author{
A. Alonso-Herrero ${ }^{1,2}$ (1) , M. Pereira-Santaella ${ }^{2}$ (D) S. García-Burillo ${ }^{3}$ (D), R. I. Davies ${ }^{4}$, F. Combes ${ }^{5}$ (iD, D. Asmus ${ }^{6,7}$ (i) A. Bunker $^{2}$, \\ T. Díaz-Santos ${ }^{8}$ (1) , P. Gandhi ${ }^{7}$ (10, O. González-Martín ${ }^{9}$ (1) , A. Hernán-Caballero ${ }^{10}$ (1) , E. Hicks $^{11}$, S. Hönig ${ }^{7}$, A. Labiano ${ }^{12}$, \\ N. A. Levenson ${ }^{13}$, C. Packham ${ }^{14}$, C. Ramos Almeida ${ }^{15,16}$ (), C. Ricci ${ }^{8,17,18}$ (1) D. Rigopoulou ${ }^{2}$, \\ D. Rosario ${ }^{19}$ (1D) E. Sani ${ }^{6}$, and M. J. Ward ${ }^{19}$ \\ ${ }^{1}$ Centro de Astrobiología (CAB, CSIC-INTA), ESAC Campus, E-28692 Villanueva de la Cañada, Madrid, Spain; aalonso@cab.inta-csic.es \\ ${ }^{2}$ Department of Physics, University of Oxford, Keble Road, Oxford OX1 3RH, UK \\ 3 Observatorio de Madrid, OAN-IGN, Alfonso XII, 3, E-28014 Madrid, Spain \\ ${ }^{4}$ Max Planck Institut fuer extraterrestrische Physik Postfach 1312, D-85741 Garching bei München, Germany \\ ${ }^{5}$ LERMA, Obs. de Paris, PSL Research Univ., Collége de France, CNRS, Sorbonne Univ., UPMC, Paris, France \\ ${ }^{6}$ European Southern Observatory, Casilla 19001, Santiago 19, Chile \\ ${ }^{7}$ Department of Physics \& Astronomy, University of Southampton, Hampshire SO17 1BJ, Southampton, UK \\ ${ }^{8}$ Núcleo de Astronomía de la Facultad de Ingeniería, Universidad Diego Portales, Av. Ejército Libertador 441, Santiago, Chile \\ ${ }^{9}$ Instituto de Radioastronomía y Astrofísica (IRyA-UNAM), 3-72 (Xangari), 8701, Morelia, Mexico \\ ${ }^{10}$ Departamento de Astrofísica y CC. de la Atmósfera, Facultad de CC. Físicas, Universidad Complutense de Madrid, E-28040 Madrid, Spain \\ ${ }^{11}$ Department of Physics and Astronomy, University of Alaska Anchorage, AK 99508-4664, USA
${ }^{2}$ Centro de Astrobiología (CAB, CSIC-INTA), Carretera de Torrejón a Ajalvir, E-28850 Torrejón de Ardoz, Madrid, Spain \\ ${ }^{13}$ Space Telescope Science Institute, 3700 San Martin Drive, Baltimore, MD 21218, USA
Department of Physics and Astronomy, University of Texas at San Antonio, 1 UTSA Circle, San Antonio, TX 78249, USA \\ ${ }_{16}$ Instituto de Astrofísica de Canarias, Calle vía Láctea, s/n, E-38205 La Laguna, Tenerife, Spain \\ ${ }^{16}$ Departamento de Astrofísica, Universidad de La Laguna, E-38205 La Laguna, Tenerife, Spain \\ ${ }^{17}$ Chinese Academy of Sciences South America Center for Astronomy, Camino El Observatorio 1515, Las Condes, Santiago, Chile \\ ${ }^{18}$ Kavli Institute for Astronomy and Astrophysics, Peking University, Beijing 100871, People's Republic of China \\ ${ }^{19}$ Centre for Extragalactic Astronomy, Durham University, South Road, Durham DH1 3LE, UK \\ Received 2018 February 19; revised 2018 April 9; accepted 2018 April 11; published 2018 June 1
}

\begin{abstract}
We present ALMA Band $6{ }^{12} \mathrm{CO}(2-1)$ line and rest-frame $232 \mathrm{GHz}$ continuum observations of the nearby Compton-thick Seyfert galaxy NGC 5643 with angular resolutions 0". 11-0". 26 (9-21 pc). The CO(2-1) integrated line map reveals emission from the nuclear and circumnuclear region with a two-arm nuclear spiral extending $\sim 10^{\prime \prime}$ on each side. The circumnuclear $\mathrm{CO}(2-1)$ kinematics can be fitted with a rotating disk, although there are regions with large residual velocities and/or velocity dispersions. The $\mathrm{CO}(2-1)$ line profiles of these regions show two different velocity components. One is ascribed to the circular component and the other to the interaction of the AGN outflow, as traced by the $[\mathrm{O}$ III] $\lambda 5007 \AA$ emission, with molecular gas in the disk a few hundred parsecs from the AGN. On nuclear scales, we detected an inclined $\mathrm{CO}(2-1)$ disk (diameter 26 pc, FWHM) oriented almost in a north-south direction. The $\mathrm{CO}(2-1)$ nuclear kinematics can be fitted with a rotating disk that appears to be tilted with respect to the large-scale disk. There are strong non-circular motions in the central 0 !" $2-0$ !" 3 with velocities of up to $110 \mathrm{~km} \mathrm{~s}^{-1}$. In the absence of a nuclear bar, these motions could be explained as radial outflows in the nuclear disk. We estimate a total molecular gas mass for the nuclear disk of $M\left(\mathrm{H}_{2}\right)=1.1 \times 10^{7} M_{\odot}$ and an $\mathrm{H}_{2}$ column density toward the location of the AGN of $N\left(\mathrm{H}_{2}\right) \sim 5 \times 10^{23} \mathrm{~cm}^{-2}$, for a standard CO-to- $\mathrm{H}_{2}$ conversion factor. We interpret this nuclear molecular gas disk as the obscuring torus of NGC 5643 as well as the collimating structure of the ionization cone.
\end{abstract}

Key words: galaxies: active - galaxies: individual (NGC 5643) - galaxies: Seyfert - molecular data

\section{Introduction}

The key piece of the Unified Model for active galactic nuclei (AGNs) is the torus of dust and molecular gas that obscures the direct view of the AGNs along certain lines of sight and explains the observational properties of AGNs (Antonucci 1993). ALMA observations detected the torus in the nearby Seyfert NGC 1068 in both cold dust and molecular line emission (Gallimore et al. 2016; García-Burillo et al. 2016; Imanishi et al. 2018). The measured torus diameter at different ALMA molecular transitions and dust continuum (7-13 pc) is approximately a factor of two larger than the size derived from the modeling of the nuclear unresolved near-to-mid-infrared emission (Alonso-Herrero et al. 2011; Ramos Almeida et al. 2011; Ichikawa et al. 2015) and mid-infrared interferometry (Tristram et al. 2009; Burtscher et al. 2013; López-Gonzaga et al. 2014). Therefore, the full extent of the torus is larger than that of the warm dust probed by the near and mid-infrared continuum. Indeed, Fuller et al. (2016) showed that when including data to $\sim 40 \mu \mathrm{m}$ the parameterization of the torus is indeed altered, further demonstrating the need for a broad wavelength sampling of the torus emission.

Cold molecular gas is detected in the nuclear and circumnuclear regions of nearby Seyfert galaxies on physical scales of tens to hundreds of parsecs (e.g., Tacconi et al. 1994; Schinnerer et al. 2000; García-Burillo et al. 2005, 2014; Krips et al. 2007; Sani et al. 2012; Combes et al. 2013; Izumi et al. 2016; Lin et al. 2016; Zschaechner et al. 2016; Salak et al. 2017). This gas is believed to be associated with the AGN fueling processes. Indeed, in local AGNs the hot ( $T \sim 1000-2000 \mathrm{~K}$; see Mouri 1994) molecular gas, as traced 
by the near-IR rovibrational $2.12 \mu \mathrm{m} 1-0 \mathrm{~S}(1) \mathrm{H}_{2}$ line, is more centrally concentrated than in nonactive galaxies and believed to be related to the Unified Model obscuring torus (Hicks et al. 2009, 2013).

A natural consequence of the accumulation of material in the central regions of active galaxies is the presence of nuclear $(<100 \mathrm{pc})$ ongoing/recent star formation activity (e.g., Davies et al. 2007; Esquej et al. 2014). Since the nuclear star formation rate is found to be correlated with the velocity dispersion of the nuclear hot molecular gas disks (Hicks et al. 2013), then the nuclear molecular gas disks could be maintained by inflows of material into the nuclear region and/or by intense, short-lived nuclear star formation through stellar feedback.

We have started several ALMA programs to observe the cold molecular gas in the nuclear and circumnuclear regions of a hard X-ray selected sample of nearby Seyfert galaxies drawn from the X-ray Swift/BAT all sky 70 month catalog (Baumgartner et al. 2013). The goal of these ALMA programs is to understand the connections between the cold and hot molecular gas, the AGN torus and nuclear/circumnuclear star formation activity in AGNs. As explained above, the nearinfrared $\mathrm{H}_{2}$ lines provide useful information about the morphology and kinematics of the hot molecular gas in the nuclear regions of Seyfert galaxies. However, they only probe a small fraction of the total molecular gas fraction in galaxies (see, e.g., Dale et al. 2005).

In this work, we present ALMA Band 6 continuum and ${ }^{12} \mathrm{CO}(2-1)$ observations of the nearby $\left(D=16.9 \mathrm{Mpc}, 1^{\prime \prime}=\right.$ 81.9 pc) Seyfert 2 galaxy NGC 5643. Although this galaxy is classified as an $\mathrm{SAB}(\mathrm{rs}) \mathrm{c}$, it has a large-scale stellar bar identified in the near-infrared (Jungwiert et al. 1997; Mulchaey et al. 1997). It is a Compton-thick (see Guainazzi et al. 2004; Annuar et al. 2015; Ricci et al. 2015) galaxy with a moderate intrinsic X-ray luminosity $\left(L_{2-10 \mathrm{keV}} \sim 10^{42} \mathrm{erg} \mathrm{s}^{-1}\right)$. Optical broad lines in polarized light have not been detected in this galaxy (Ramos Almeida et al. 2016). These authors explained it as a combination of its Compton-thick nature and relatively low AGN luminosity or different properties of the scattering material.

The $2.12 \mu \mathrm{m} \mathrm{1-0} \mathrm{S(1)} \mathrm{H}_{2}$ hot molecular gas in the central region of NGC 5643 shows anomalous kinematics in an area at about $2^{\prime \prime}$ northeast of the nucleus with high velocity dispersion (Davies et al. 2014). This might be a signature of the presence of molecular gas being excited near the edge of the ionization cone traced by the optical [O III] $\lambda 5007 \AA$ line (Simpson et al. 1997). The radio emission of NGC 5643 shows an almost east-west orientation extending for about $30^{\prime \prime}$ on both sides of the nucleus (Morris et al. 1985; Leipski et al. 2006). This radio structure appears to be impacting on the disk on the east side of the galaxy producing positive feedback as revealed by the presence of H II regions at approximately $5^{\prime \prime}$ and $10^{\prime \prime}$ east of the nucleus (Cresci et al. 2015). The soft X-ray emission is also extended and mostly detected in the east side of the galaxy following the [O III] emission (Bianchi et al. 2006; Gómez-Guijarro et al. 2017).

The paper is organized as follows. Section 2 describes the ALMA observations as well as archival optical integral field spectroscopy. In Sections 3 and 4, we present the analysis of the ALMA $\mathrm{CO}(2-1)$ morphology and kinematics of the circumnuclear and nuclear region of NGC 5643, respectively. In Section 5, we discuss the results and give our conclusions.

\section{Observations}

\subsection{ALMA Band 6 Observations}

We obtained Band 6 ALMA observations of NGC 5643 on 2016 December 24 and 2017 July 18 using the $12 \mathrm{~m}$ array in compact (baselines between 15 and $492 \mathrm{~m}$ ) and extended (baselines between 17 and $3700 \mathrm{~m}$ ) configurations through the project 2016.1.00254.S (PI: A. Alonso-Herrero). The on-source integrations times were 11 and 36 minutes, respectively. We defined two spectral windows of $1.875 \mathrm{GHz}$ bandwidth $\left(3.9 \mathrm{MHz} \sim 5 \mathrm{~km} \mathrm{~s}^{-1}\right.$ channels), namely, one at $\sim 228 \mathrm{GHz}$ at the observed frequency of the ${ }^{12} \mathrm{CO}(2-1)$ transition and the other at observed frequency of $\sim 230 \mathrm{GHz}$ to measure the submillimeter continuum.

We calibrated the data using the ALMA reduction software CASA (v.4.7; McMullin et al. 2007). For both configurations, we used J1427-4206 as the bandpass and phase calibrator. The amplitude was calibrated using J1427-4206 for the extended configuration assuming a flux density of $1.92 \mathrm{Jy}$ at $228.6 \mathrm{GHz}$ and a spectral index of -0.59 . For the compact configuration, we used Callisto as the amplitude calibrator assuming the Butler-JPL-Horizons 2012 model.

For the $\mathrm{CO}(2-1)$ spectral window, we subtracted the continuum (rest-frame $232 \mathrm{GHz}$ ) in the $u v$ plane by fitting the continuum with a constant in the line-free channels. Then, we combined and cleaned the data from the two configurations using the CASA clean task. The output frequency reference frame was set to the kinematic local standard of rest (LSRK).

We produced three sets of cleaned data For the first one we used natural weight to emphasize the large-scale emission of NGC 5643. For the other two we used the Briggs weighting (Briggs 1995), with robustness parameters of $b=0.8$ and $b=-0.5$ to increase sequentially the angular resolution at slightly decreased sensitivity. For the $b=-0.5$ data set we kept the original spacings of the frequency channel, whereas for the $b=0.8$ set we rebinned the data by a factor of 3 in frequency to increase the signal-to-noise ratio. This resulted in an approximate resolution in velocities of $5 \mathrm{~km} \mathrm{~s}^{-1}$ and a $\mathrm{CO}(2-1)$ FWHM of 0 " $16 \times 0$ ". 11 with a beam position angle $\left(\mathrm{PA}_{\text {beam }}\right)$ of $-67^{\circ}$ for the $b=-0.5$ data set, and $15 \mathrm{~km} \mathrm{~s}^{-1}$ and an FWHM of 0 ". $26 \times 0$ ". 17 with $\mathrm{PA}_{\text {beam }}=-58^{\circ}$ for the $b=0.8$ data set. For the continuum images, the beams FWHMs are 0 ". $16 \times 0$ ". 10 at $\mathrm{PA}_{\text {beam }}=-65^{\circ}$ for $b=-0.5$ and and $0 . .23 \times 0$." 13 at $\mathrm{PA}_{\text {beam }}=-68^{\circ}$ for $b=0.8$. The pixel sizes were set to $0 .^{\prime \prime} 03$ and 0 ." 04 to properly sample the beam sizes, for the $b=-0.5$ and $b=0.8$ data sets, respectively. The achieved $1 \sigma$ sensitivities are $\sim 1.2$ and $\sim 0.5$ mJy beam $^{-1}$ channel $^{-1}$ in the $\mathrm{CO}(2-1)$ cubes and $\sim 76$ and $\sim 37 \mu \mathrm{Jy}$ beam $^{-1}$ in the continuum images for the $b=-0.5$ and $b=0.8$ data sets, respectively. For all three data cubes we produced maps of the $\mathrm{CO}(2-1)$ integrated intensity, mean-velocity field, and velocity dispersion. For all of these maps we used pixels at all frequencies with detections $>3 \sigma$. We applied the primary beam $\left(\mathrm{FWHM}=27^{\prime \prime}\right)$ correction to the data.

In Figure 1 (lower panel), we show the integrated $\mathrm{CO}(2-1)$ molecular line map created with the natural-weight data. The field of view (FoV) is approximately 39 ". $9 \times$ 19 !.9 $(\sim 3.3 \mathrm{kpc} \times 1.6 \mathrm{kpc})$ with an angular resolution of 0 . $26 \times 0$." 17 at $\mathrm{PA}_{\text {beam }}=-69^{\circ} .6$. We note that the primary beam of the observations is smaller than this FoV. However, most of the detected emission is well inside the FoV of the primary beam. The line map shows a bright nuclear source, a nuclear two-arm spiral already seen in optical to near-IR color 

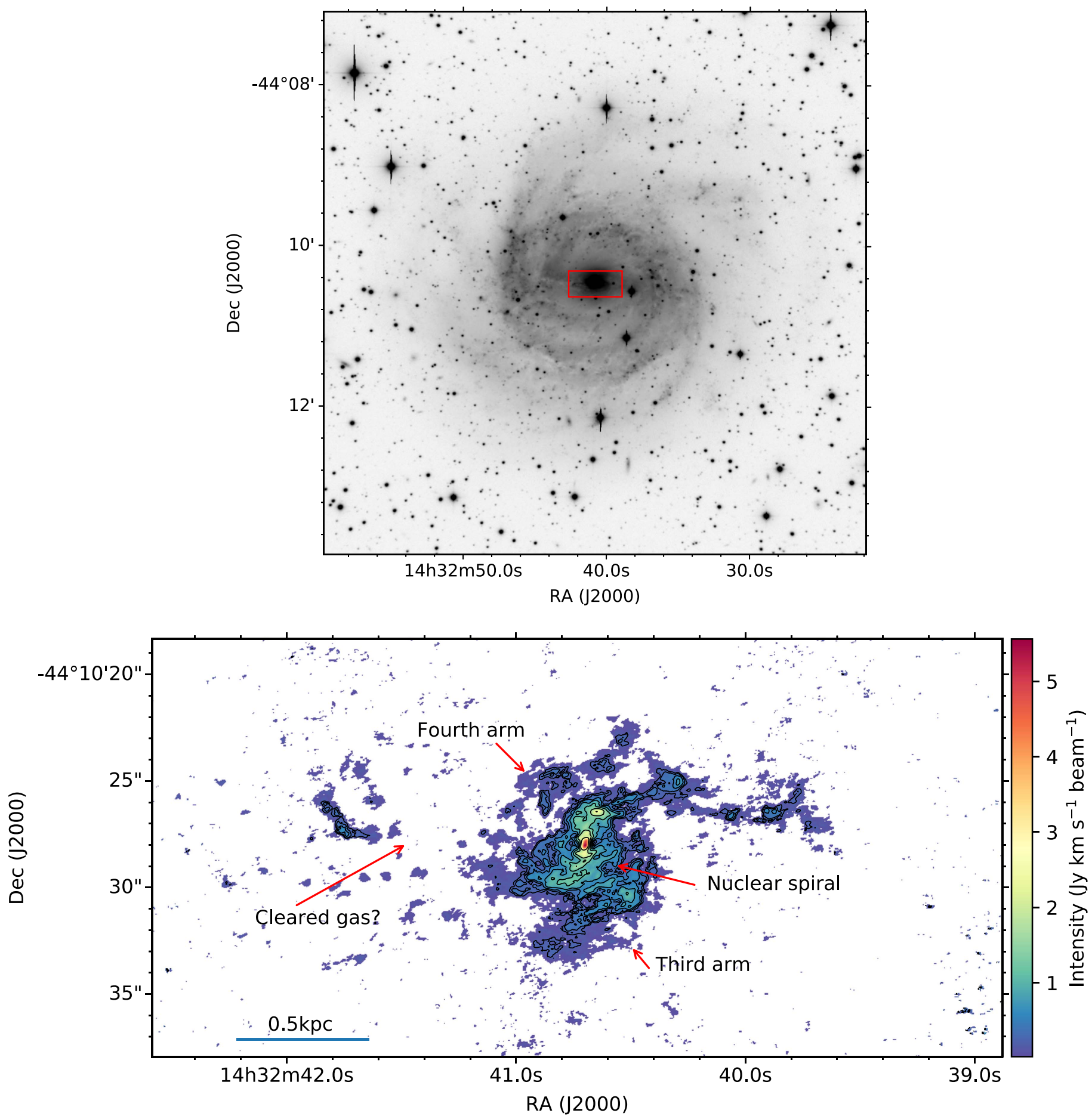

Figure 1. Top panel: optical image of NGC 5643 from the Carnegie-Irvine Galaxy Survey (Ho et al. 2011). The red rectangle marks the approximate size of the ALMA map below. Lower panel: ALMA CO(2-1) natural-weighted integrated intensity map of NGC 5643 produced with a $3 \sigma$ clipping and shown in a linear scale to emphasize the large-scale emission. The contours are shown in a logarithmic scale with the first contour at $0.2 \mathrm{Jy} \mathrm{km} \mathrm{s}^{-1}$ beam ${ }^{-1}$ and the last contour at $2.5 \mathrm{Jy} \mathrm{km} \mathrm{s}^{-1}$ beam $^{-1}$. The FoV of the image is approximately $39^{\prime \prime} 9 \times 19^{\prime \prime} 9(\sim 3.3 \mathrm{kpc} \times 1.6 \mathrm{kpc})$ and the angular resolution $00^{\prime \prime} 26 \times 00^{\prime \prime} 17$ at $\mathrm{PA}_{\mathrm{beam}}=-69^{\circ} 6$. We applied the primary beam $\left(27^{\prime \prime}\right)$ correction to the data.

maps as a dusty nuclear spiral extending for several arcseconds (Quillen et al. 1999; Martini et al. 2003; Davies et al. 2014) as well as emission in the spiral arms on larger scales. We measure a $\mathrm{CO}(2-1)$ line intensity over a region $40^{\prime \prime} \times 40^{\prime \prime}$ of $480 \mathrm{Jy} \mathrm{km} \mathrm{s}^{-1}$. For comparison the single-dish measurement for a beam size of $30^{\prime \prime}$ is $912 \pm 60 \mathrm{Jy} \mathrm{km} \mathrm{s}^{-1}$ (Monje et al. 2011). Thus, we recovered approximately $50 \%$ of the single-dish observation flux that is typical of this kind of comparison.

\subsection{Archival VLT/MUSE Integral Field Spectroscopy}

NGC 5643 was observed with the Multi Unit Spectroscopic Explorer (MUSE; Bacon et al. 2010) on the Very Large 

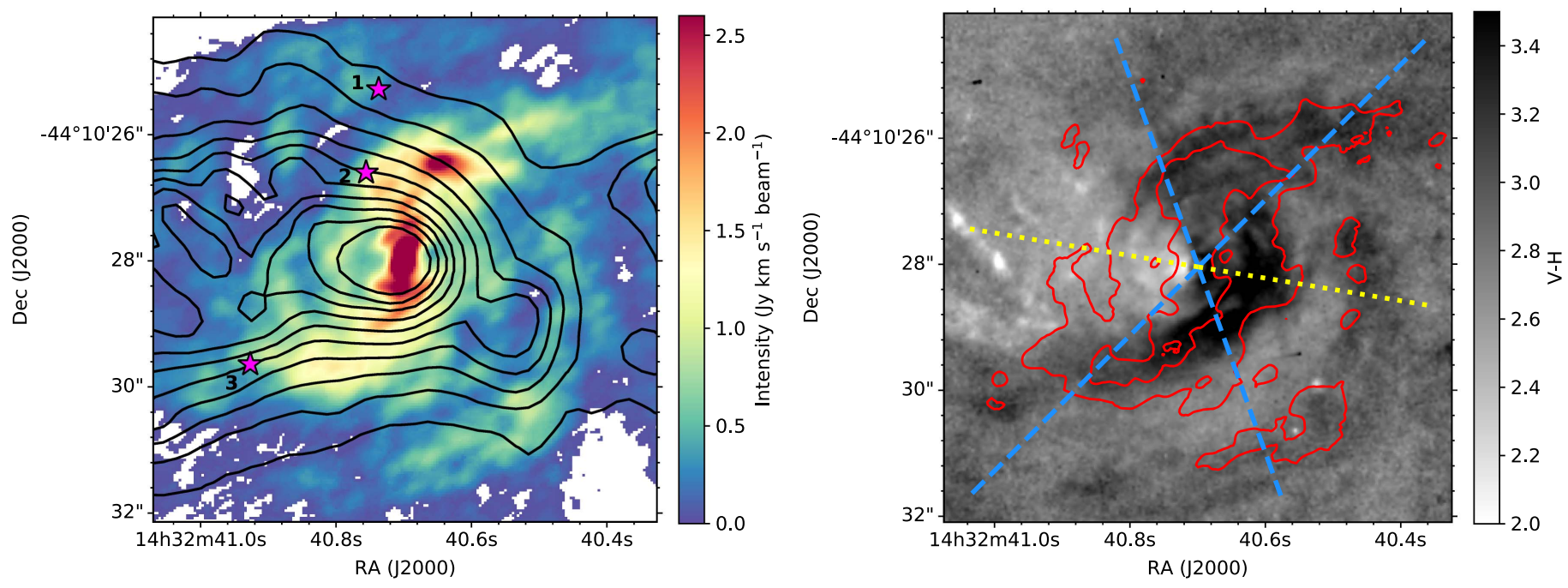

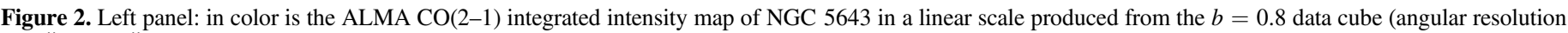

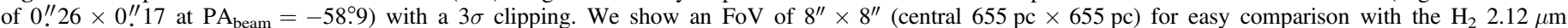

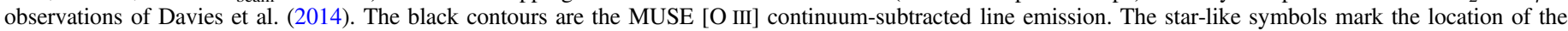

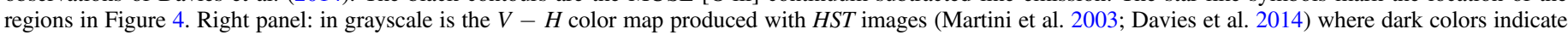

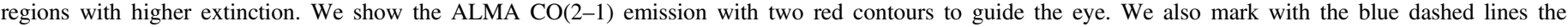

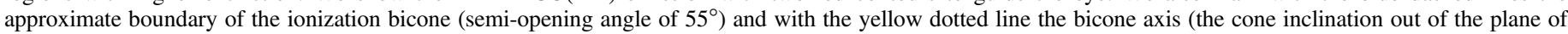
the sky toward Earth is $\sim 25^{\circ}$, and $\sim 40^{\circ}$ with respect to the galaxy disk plane), as modeled by Fischer et al. (2013).

Telescope (VLT) of the European Southern Observatory (ESO) on 2015 May 12. These observations were part of the program 095.B-0532(A) (PI: Carollo). We downloaded the pipeline processed data cube from the ESO data archive. We estimate a seeing of $\sim 0$." 5 from the foreground stars in the FoV. Four dithered exposures of $875 \mathrm{~s}$ were taken and later combined into a single cube by the pipeline. This final combined cube covers a $1^{\prime} \times 1^{\prime}$ FoV with a pixel size of 0 .' 2 . The observed spectral range is $475-935 \mathrm{~nm}$ and the spectral resolution varies between $R=\lambda / \Delta \lambda_{\text {FWHM }} \sim 1770$ at $480 \mathrm{~nm}$ and $R=3590$ at $930 \mathrm{~nm}$.

We produced line emission maps and velocity fields of the [O III] $\lambda 4959$ and $\lambda 5007$ doublet (the latter will be referred to as [O III] henceforth), the [N II] $\lambda 6549$ and $\lambda 6583$ doublet, and $\mathrm{H} \alpha$ at $6563 \AA$. We fitted a single Gaussian profile to each of these transitions in each spaxel. The underlying local continuum was approximated by a straight line. The flux ratio of the doublets was fixed to the expected theoretical ratio of 0.35 and 0.34 for the [O III] and [N II] doublets, respectively. The [N II] and $\mathrm{H} \alpha$ transitions were fitted simultaneously.

Finally, we aligned the MUSE images using the peak of the continuum images to match the coordinates of the ALMA continuum peak at rest-frame $232 \mathrm{GHz}(1.3 \mathrm{~mm})$ R.A. $(\mathrm{J} 2000)=14^{\mathrm{h}} 32^{\mathrm{m}} 40^{\mathrm{s}} .70$ and decl. $(\mathrm{J} 2000)=-44^{\circ} 10^{\prime} 27^{\prime \prime} \cdot 9$.

\section{Circumnuclear Region}

In this section, we focus on the $\mathrm{CO}(2-1)$ emission in the circumnuclear region of NGC 5643 over a FoV of $8^{\prime \prime} \times 8^{\prime \prime}$ (central $655 \mathrm{pc} \times 655 \mathrm{pc}$ ) and use the $b=0.8$ data set. We selected this region to compare the cold molecular gas emission with the VLT/SINFONI integral field spectroscopy study of this galaxy done by Davies et al. (2014) with this FoV. Nevertheless, as can be seen from Figure 1 (lower panel), most of the ALMA $\mathrm{CO}(2-1)$ emission $(\sim 80 \%)$ arises from this region. Based on the kinematics of the hot molecular gas line $\mathrm{H}_{2}$ at $2.12 \mu \mathrm{m}$ Davies et al. (2014) found evidence of outflowing material on scales $1^{\prime \prime}-2^{\prime \prime}$ from the nucleus. This material appears to be at the edge of the eastern ionization cone probed by the [O III] emission observed with the Hubble Space Telescope (HST) by Simpson et al. (1997) and more recently with MUSE by Cresci et al. (2015).

\subsection{Morphology}

Figure 2 (left panel) shows in color the $\mathrm{CO}(2-1)$ velocityintegrated intensity map. The brightest emission comes from the central region (see Section 4.1 for a detailed discussion) from which the nuclear two-arm spiral structure is clearly connected. The spiral structure extends for more than $10^{\prime \prime}$ on both sides of the galaxy (see the lower panel of Figure 1), although the east side spiral arm shows a region void of $\mathrm{CO}(2-1)$ emission gas. We will come back to this in Section 5. The spiral arms are oriented in an almost east-west direction as is the large-scale stellar bar (see, e.g., Jungwiert et al. 1997; Mulchaey et al. 1997; and also the upper panel of Figure 1). There is also $\mathrm{CO}(2-1)$ emission in a third spiral-like structure in the circumnuclear region located to the west of the nucleus. We marked this third arm in Figure 1 (lower panel). This structure was first detected in $\mathrm{H}_{2}$ at $2.12 \mu \mathrm{m}$ emission by Davies et al. (2014). They interpreted it as a transient structure resulting from a recent perturbation to the galaxy. Alternatively, it might be attributed to the far side of the ionization cone (that is, the counter-cone) where it is bisecting the galaxy and perturbing the ambient gas. In the nuclear region, the tips of the nuclear spiral arms connect with dusty arc-like structures $\sim 1$ ". 4 in size. In the innermost region, a disk-like structure ( $\sim 0$ " 6 diameter) in the north-south direction is detected. These will be discussed in detail in Section 4.

We superimposed on the $\mathrm{CO}(2-1)$ map in Figure 2 as black contours our MUSE continuum-subtracted [O III] line emission map (see Section 2.2). We note that this new MUSE data set was observed under better seeing conditions than the Cresci et al. (2015) one (0." 5 versus $0 . " 88)$. As discussed by these authors and also seen in our figure, the ionization cone traced by the [O III] emission is more clearly seen to the east of the nucleus due to obscuration produced by the host galaxy in the 
west direction. However, the MUSE [O III] image also traces the counter-cone emission on scales of a few arcseconds to the west of the nucleus (Cresci et al. 2015). This comparison shows that most of the $\mathrm{CO}(2-1)$ bright emission seems to avoid the regions of ionized [O III] gas. This would suggest that the $\mathrm{CO}(2-1)$ emission is in the plane of the galaxy, whereas most of the [O III] emission is outflowing perpendicular to the accretion disk and intercepts the host galaxy disk, since the ionization cone has an inclination of approximately $40^{\circ}$ with respect to the galaxy disk (see also Fischer et al. 2013 and Section 3.2). Similar situations of the molecular gas avoiding the ionization cone have been reported in the literature for NGC 4253 and M51 (see Krause et al. 2007; Querejeta et al. 2016, respectively).

The right panel of Figure 2 is the $H S T V-H$ color map (in grayscale) from Martini et al. (2003) and Davies et al. (2014). We overlaid on this image two contours showing the $\mathrm{CO}(2-1)$ integrated emission (that of the left panel) to guide the eye. Clearly the nuclear spiral structure outlined by the nearly horizontal dust lanes (dark $V-H$ regions) entering the nuclear region is coincident with the $\mathrm{CO}(2-1)$ molecular gas emission. The third spiral-like structure is traced by both the $\mathrm{CO}(2-1)$ cold gas emission and the dust lanes. Also there is evidence that this third spiral structure might have a fainter counterpart (i.e., a fourth arm) on the east side of the galaxy again seen in both dust and cold molecular gas (see also Figure 1 for a larger FoV).

\subsection{Kinematics}

We used the ${ }^{3 \mathrm{D}}$ BAROLO code (Di Teodoro \& Fraternali $2015)$ to model the $\mathrm{CO}(2-1)$ kinematics of the central $8^{\prime \prime} \times 8^{\prime \prime}$ region of NGC 5643 using the ALMA $b=0.8$ data cube. ${ }^{3 \mathrm{D}}$ BAROLO was designed to fit simple disk models using 3D tilted rings to a variety of emission line data, including ALMA data cubes. The fit is done in two steps. During the first step for each ring, ${ }^{3 \mathrm{D}} \mathrm{BAROLO}$ fits the free parameters, namely, the kinematic center, the systemic velocity, the disk inclination and PA of the major axis, the scale height of the disk, the circular velocity, and the velocity dispersion. The second step fixes the kinematic center and systemic velocity to the mean fitted values from the first iteration and finds the best solution for the other free parameters. ${ }^{3 \mathrm{D}} \mathrm{BAROLO}$ also allows the user to fix any of the above parameters during the first iteration. After fitting the data cube, ${ }^{3 \mathrm{D}} \mathrm{BAROLO}$ creates, among other products, a map of the observed velocity-integrated intensity (0th moment), a map of the observed mean-velocity field (1st moment) and velocity dispersion field (2nd moment), as well as the same maps for the best-fit model.

We first run ${ }^{3 \mathrm{D}} \mathrm{BAROLO}$ on the $\mathrm{CO}(2-1)$ data cube using the disk PA and inclination derived from the near-infrared stellar kinematics by Davies et al. (2014) over the same FoV allowing for relatively small variations (approximately $\pm 20^{\circ}$ for the disk PA and $\pm 10^{\circ}$ for the disk inclination). After the first run we fixed the disk PA and inclination to the average fitted values of $\mathrm{PA}_{\text {disk }}=320^{\circ}$ and $i=35^{\circ}$, which are compatible with those inferred from the stellar kinematics (Davies et al. 2014). We also fixed the systemic velocity to $v_{\mathrm{sys}}=1194 \mathrm{~km} \mathrm{~s}^{-1}$ (mean value of the first run) and rerun ${ }^{3 \mathrm{D}} \mathrm{BAROLO}$ to fit the observed velocity field.

The results are shown in Figure 3. The top panels are the ${ }^{3 \mathrm{D}} \mathrm{BAROLO}$ maps of the observed mean $\mathrm{CO}(2-1)$ velocity field (1st moment map, left) and velocity dispersion (2nd moment map, right). These can be compared directly with the $\mathrm{H}_{2} 2.12 \mu \mathrm{m}$ hot molecular gas maps in Figure 8 of Davies et al. (2014). Both the mean-velocity fields of the cold and hot molecular gas are similar and display a clear rotational pattern with evidence of some non-circular motions. The velocity dispersion maps of the cold and hot molecular gas show a peak at the nucleus position with a value of $\sigma(\mathrm{CO}(2-1))$ $\sim 60 \mathrm{~km} \mathrm{~s}^{-1}$. However, the $\mathrm{H}_{2}$ map shows the highest velocity dispersion value, $\sigma\left(\mathrm{H}_{2}\right) \sim 100 \mathrm{~km} \mathrm{~s}^{-1}$, at about $2^{\prime \prime}$ to the northeast of the nucleus. This peak is not as clearly seen in the map of $\mathrm{CO}(2-1)$ velocity dispersion due to low signal-to-noise ratio in that region of our map. Nevertheless, there is a region to the north-northeast of the AGN at about $3^{\prime \prime} \sim 246 \mathrm{pc}$ with a $\mathrm{CO}(2-1)$ velocity dispersion value similar to that of the nucleus (see below).

We constructed a $\mathrm{CO}(2-1)$ residual mean-velocity field map (see Figure 3, bottom right panel) by subtracting the 3D BAROLO disk model (Figure 3, bottom left panel) from the ${ }^{3 \mathrm{D}}$ BAROLO first moment map. Some of the residual velocity field seen in the central few arcseconds could be an artifact of the simple disk geometry assumed in our modeling with ${ }^{3 D}$ BAROLO. In particular, a model that accounted for the presence of a bar potential would better predict the streaming motions linked to the gas response to the bar along the leading edges of the nuclear spiral (see, for instance, Emsellem et al. 2001). However, it would not be able to reproduce the strong redshifted velocity residuals $\left(\sim 40-60 \mathrm{~km} \mathrm{~s}^{-1}\right)$ seen $\sim 2^{\prime \prime}-3^{\prime \prime}$ northeast of the AGN. This redshifted component was also observed in the velocity field of the hot molecular gas $\mathrm{H}_{2}$ at $2.12 \mu \mathrm{m}$ (Davies et al. 2014) and shows high $\mathrm{CO}(2-1)$ velocity dispersion.

To look further into the gas kinematics of regions with the large velocity residuals with respect to a simple rotating disk and/or velocity dispersions, we extracted $\mathrm{CO}(2-1)$ line profiles at three positions. The first two have redshifted components and are located approximately 2."7 north-northeast and 1".5 northeast ( $\sim 221$ and 123 pc, respectively, and see Figure 2 for the locations) from the peak of the $\mathrm{CO}(2-1)$ emission. The top and middle panels of Figure 4 show the line profiles obtained with a square aperture of 10 pixels or 0 "! 4 . Both regions clearly show two distinct velocity components, one associated with the rotation of the large-scale disk and a second one redshifted by approximately $30-45 \mathrm{~km} \mathrm{~s}^{-1}$. The morphology of the nuclear spiral structure indicates that the rotation in the disk of the galaxy is counterclockwise. Assuming that the non-circular motions seen in these regions to the northeast of the center are coplanar with the galaxy disk, then the redshifted components imply radial outward movements in the disk of the galaxy near the edge of the [O III] ionization cone (see Figures 2 and 3). The simplest explanation for the $\mathrm{CO}(2-1)$ velocity residuals would be radial movements of material that is being pushed outward in the galaxy disk by the outflowing material traced by the [O III] emission.

The third region is in the nuclear spiral at $\sim 2$ !" 9 southeast of the nucleus (see Figure 2) and presents blue velocity residuals and an excess velocity dispersion, which are explained by the presence of two velocity components (see the bottom panel of Figure 4). This location is in a region where the [O III] outflow (identified by the presence of blueshifted components) and the radio jet appear to be interacting with the disk of the galaxy (Cresci et al. 2015). We note that this $\mathrm{CO}(2-1)$ blueshifted region is not coincident with the $\mathrm{H} \alpha$ knots resulting from 

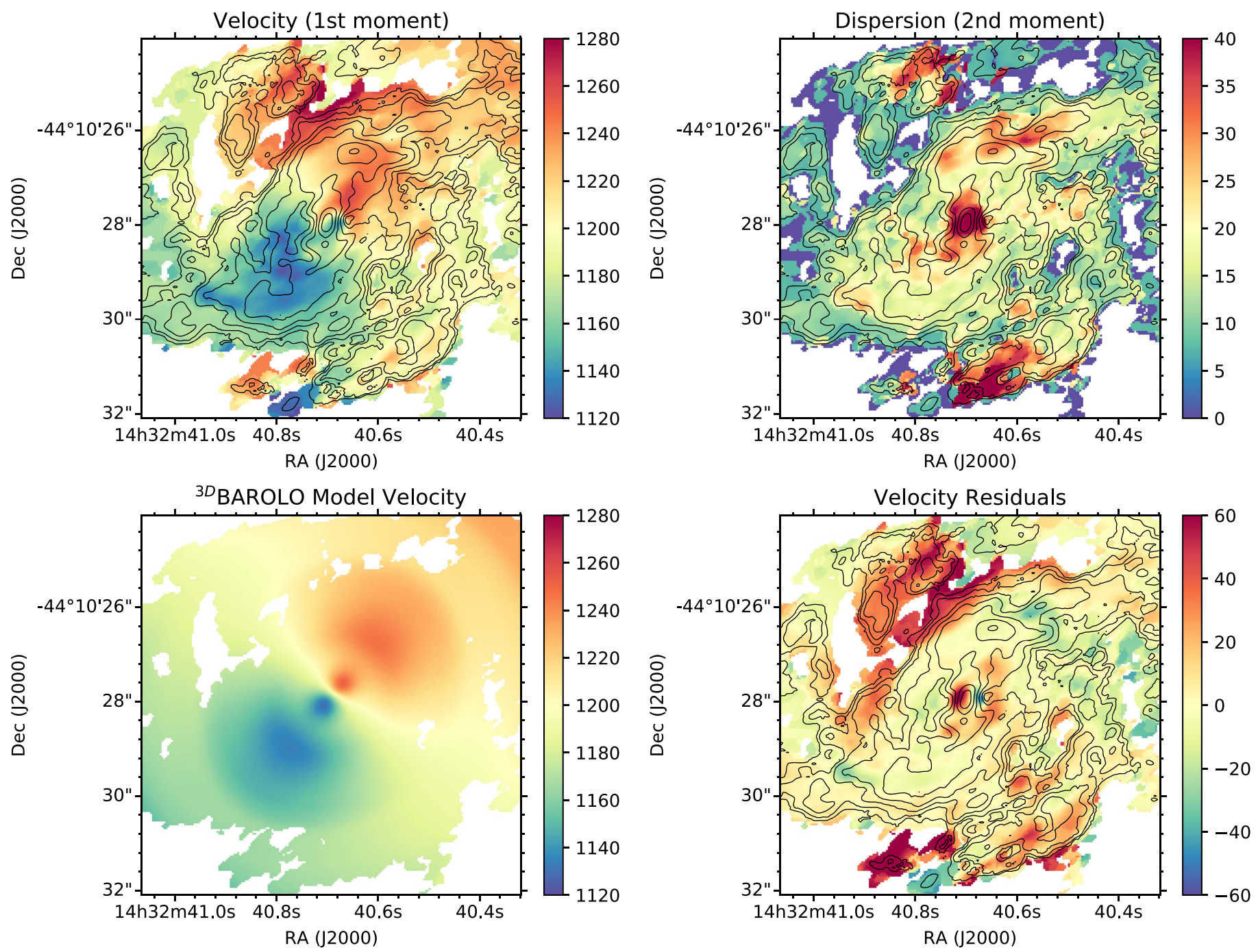

Figure 3. Modeling of the ALMA CO $(2-1)$ kinematics with ${ }^{3 \mathrm{D}} \mathrm{BAROLO}$ using the $b=0.8$ data cube (angular resolution of $00^{\prime \prime} 26 \times 00^{\prime \prime} 17$ at $\mathrm{PA}$ beam $=-58^{\circ} .9$ ) and an FoV of $8^{\prime \prime} \times 8^{\prime \prime}$ (as in Figure 2). The top left and right panels are the mean-velocity field (1st moment map) and velocity dispersion field (2nd moment map), respectively. The bottom left panel is the disk model, and the bottom right panel is the residual mean-velocity field map. The vertical color bars are velocities or velocity dispersions in $\mathrm{km} \mathrm{s}^{-1}$. The black contours are the $\mathrm{CO}(2-1)$ line intensity in a linear scale.

positive feedback induced by the jet that are located further away from the nucleus.

\section{The Nuclear Obscuring Disk}

To study the properties of the nuclear emission we use the ALMA $b=-0.5$ data cube with the highest angular resolution of 0 " $16 \times 0$ "! 11 , which corresponds to $13 \mathrm{pc} \times 9 \mathrm{pc}$ at the assumed distance of NGC 5643. As already seen from Figures 1 and 2, NGC 5643 shows bright $\mathrm{CO}(2-1)$ line emission arising from the nuclear regions.

\subsection{Morphology}

We focus here on the central 1 !" $35 \times 1$ ". 35 region which corresponds approximately to the central $110 \mathrm{pc} \times 110 \mathrm{pc}$, and encloses the arc-like structures and the nuclear disk (see the map of the $\mathrm{CO}(2-1)$ integrated intensity in Figure 5). The arc-like structures trace where the nearly horizontal nuclear spiral arms (oriented as the large-scale bar) come in (see also Figure 2). These are coincident with dusty features suggesting the direction of the inflowing material (Davies et al. 2014). The presence of these straight dust lane/gas features near the nucleus are predicted by hydrodynamical models of gas flow in a barred potential (Regan \& Mulchaey 1999; Maciejewski 2004).

The innermost region shows a disk-like morphology. This nuclear disk is fully resolved with a measured FWHM of 0 !' 32 in the approximate north-south direction and 0 ". 24 in the east-west direction that corresponds to a projected size of $26 \mathrm{pc} \times 20 \mathrm{pc}$. These values have not been corrected for the beam size of the observations. It also appears to be tilted with respect to the largescale galaxy disk (see Section 4.2). The presence of this nuclear disk was previously suggested by the dusty structure in the nuclear region (Davies et al. 2014; Cresci et al. 2015). Alternatively, we could interpret the $\mathrm{CO}(2-1)$ nuclear structure as an inner bar (see the discussion in Section 5.2).

The nuclear disk of NGC 5643 is further resolved into two peaks of $\mathrm{CO}(2-1)$ with an asymmetric structure. The rest-frame $232 \mathrm{GHz}$ continuum, which likely pinpoints the location of the $\mathrm{AGN},{ }^{20}$ is located in between the two $\mathrm{CO}(2-1)$ peaks. This

\footnotetext{
${ }^{20}$ The coordinates agree with those of the $\mathrm{H}_{2} \mathrm{O}$ maser within their reported uncertainties \pm 0 !! 2 (Greenhill et al. 2003).
} 

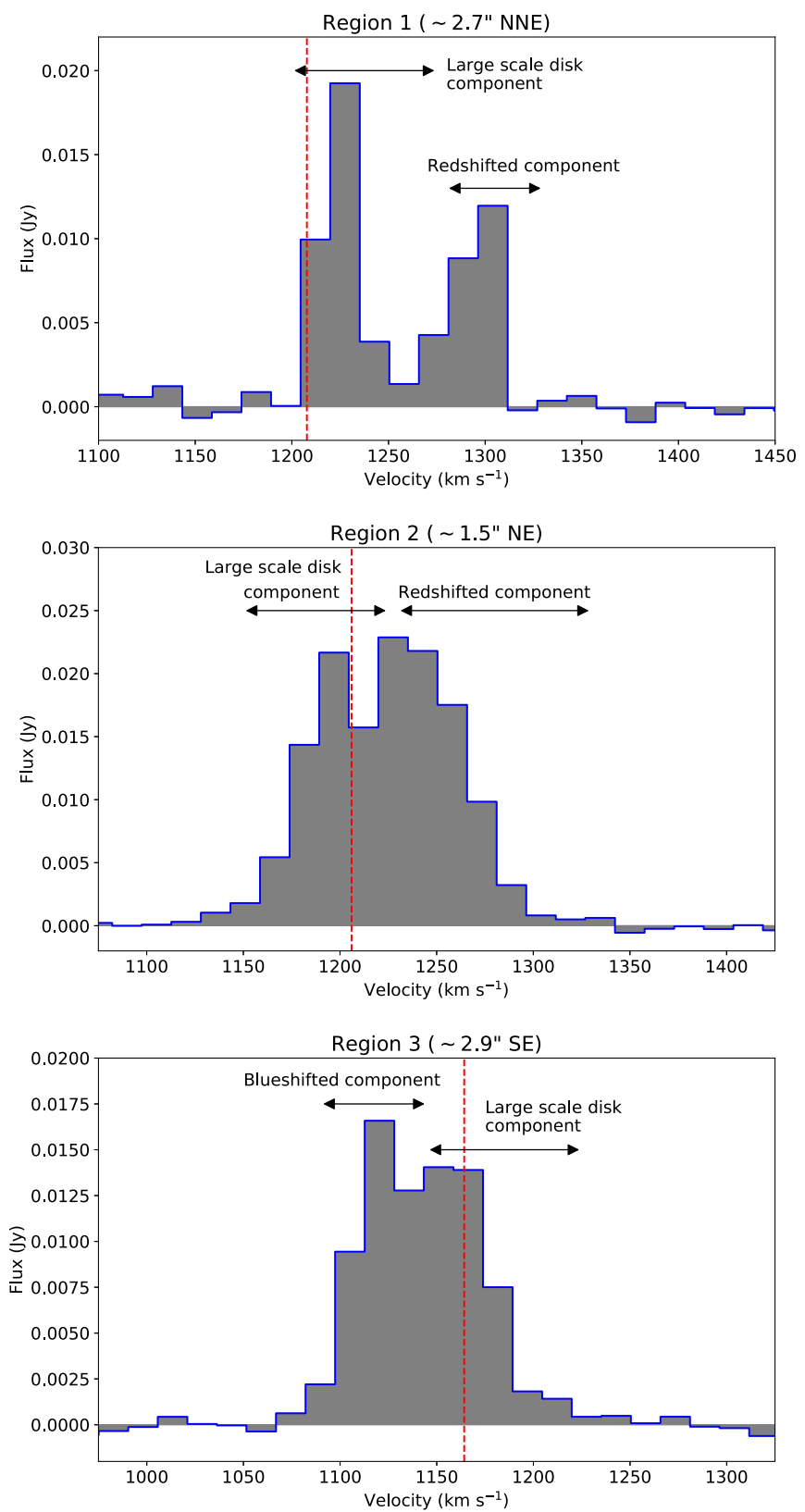

Figure 4. $\mathrm{CO}(2-1)$ line profiles extracted from the $b=0.8$ data cube at three different locations with large velocity residuals and/or large velocity dispersion. The velocity resolution of the data is approximately $15 \mathrm{~km} \mathrm{~s}^{-1}$. The vertical dashed lines are the expected disk circular velocities from the ${ }^{3 D}$ BAROLO fit. The coordinates (J2000) of the regions are as follows. Region 1: R.A. $=14^{\mathrm{h}} 32^{\mathrm{m}} 40^{\mathrm{s}} .73$ and decl. $=-44^{\circ} 10^{\prime} 25^{\prime \prime} \cdot 2$, Region 2: R.A. $=14^{\mathrm{h}}$ $32^{\mathrm{m}} 40^{\mathrm{s}} .75$ and decl. $=-44^{\circ} 10^{\prime} 26^{\prime \prime} 6$, and Region 3: R.A. $=14^{\mathrm{h}} 32^{\mathrm{m}} 40^{\mathrm{s}} .93$ and decl. $=-44^{\circ} 10^{\prime} 29^{\prime \prime} 6$.

kind of lopsided gas morphology is the characteristic $m=1$ mode predicted by numerical simulations of gas dynamics on scales of a few parsecs from an accreting supermassive black hole and that is due to angular momentum transfer (Hopkins \& Quataert 2010). However, we cannot rule out the possibility that the two $\mathrm{CO}(2-1)$ peaks reflect different gas excitation conditions in the nuclear region.

As can be see from Figure 5, the $232 \mathrm{GHz}(1.3 \mathrm{~mm})$ continuum emission is produced by a compact source, although some resolved emission seems to be present. To quantify this, we modeled the continuum in the $u v$ plane using the UVMULTIFIT library (Martín-Vidal et al. 2014) with a
Gaussian function. The best fit produced a continuum flux of $1.8 \pm 0.2 \mathrm{mJy}$, with a deconvolved size (FWHM) along the major axis of $0.20 \pm 0$. 04 and a size along the minor axis of $0.11 \pm 0 . " 05$ at $\mathrm{PA}=210^{\circ} \pm 20^{\circ}$. The orientation of the continuum extended emission would appear to follow the orientation of the inner contours of the MUSE [O III] emission. It could thus be interpreted as either polar dust emission or synchrotron emission or both. We note that radio emission in this direction is, however, observed both to the east and west of the AGN position (Morris et al. 1985; Leipski et al. 2006). Unfortunately, the angular resolution of these radio observations $\left(\sim 1^{\prime \prime}-1\right.$ !" 5$)$ does not allow us to make any further comparisons with the ALMA continuum emission.

\subsection{Kinematics}

Figure 6 (left panel) shows the mean-velocity field (1st moment map derived with $\left.{ }^{3 \mathrm{D}} \mathrm{BAROLO}\right)$ of the $\mathrm{CO}(2-1)$ molecular gas in the nuclear region. A comparison with the larger-scale $\mathrm{CO}(2-1)$ kinematics shown in Figure 3 suggests that the nuclear disk might be tilted with respect to the host galaxy disk. We used again ${ }^{3 \mathrm{D}} \mathrm{BAROLO}$ to construct a disk model to fit the kinematics of the nuclear region. We first fitted a simple isophotal model with the IRAF ellipse task to the nuclear $\mathrm{CO}(2-1)$ emission (see Figure 5, right panel). The aim is to provide ${ }^{3 \mathrm{D}} \mathrm{BAROLO}$ with initial values of the nuclear disk PA and inclination. From the outer isophotes of the nuclear disk (central $\sim 0$ ". 6 ) we can see that the nuclear disk is almost in a north-south orientation (PA $\sim 354^{\circ}$ ) and the measured ellipticity $(\epsilon=0.6)$ implies a relatively high inclination.

We followed the same procedure as explained in Section 3.2 to model the kinematics. For the nuclear disk we fixed the kinematic center to the position of the continuum peak. To derive the final ${ }^{3 \mathrm{D}} \mathrm{BAROLO}$ model we fixed the nuclear disk geometry to $\mathrm{PA}=355^{\circ}$ and $i=60^{\circ}$, and the systemic velocity to $v_{\mathrm{sys}}=1198 \mathrm{~km} \mathrm{~s}^{-1}$. These were the average values derived when running ${ }^{3 \mathrm{D}} \mathrm{BAROLO}$ leaving these parameters as well as the circular velocity and velocity dispersion free. We note that the inclination of the nuclear disk is in good agreement with the inclination of ionization cone derived by Fischer et al. (2013) from the modeling of the narrow-line region (NLR) kinematics, $i_{\text {cone }}=25^{\circ}$. If the cone is perpendicular to the collimating nuclear disk then this is equivalent to a nuclear disk inclination of $65^{\circ}$. Finally, the detection of an $\mathrm{H}_{2} \mathrm{O}$ megamaser (Greenhill et al. 2003) implies a highly inclined view to the AGN.

We show the ${ }^{3 \mathrm{D}} \mathrm{BAROLO}$ model and the residual meanvelocity field in Figure 6 (middle and right panels). Since the nuclear disk appears to be seen almost edge-on we should not see velocity residuals along the minor axis of the nuclear disk if the velocity field was perfectly circular. The residuals are thus indicative of the presence of non-circular motions in the nuclear disk. As can be seen from this figure, near the AGN peak there are redshifted residuals to the east and blueshifted residuals to the west. Outside the nuclear disk the residuals are related to the velocity field of the larger-scale disk of the galaxy, which has a different inclination and position angle, as discussed in Section 3.2.

To account for the non-circular motions seen in the nuclear disk, we rerun ${ }^{3 \mathrm{D}} \mathrm{BAROLO}$ including a radial velocity component with the same assumptions for the rotating disk as above. An inspection of Figure 7 reveals that the model with the extra radial velocity component better reproduces the observed velocity field with the residuals in the region of the nuclear disk being of 

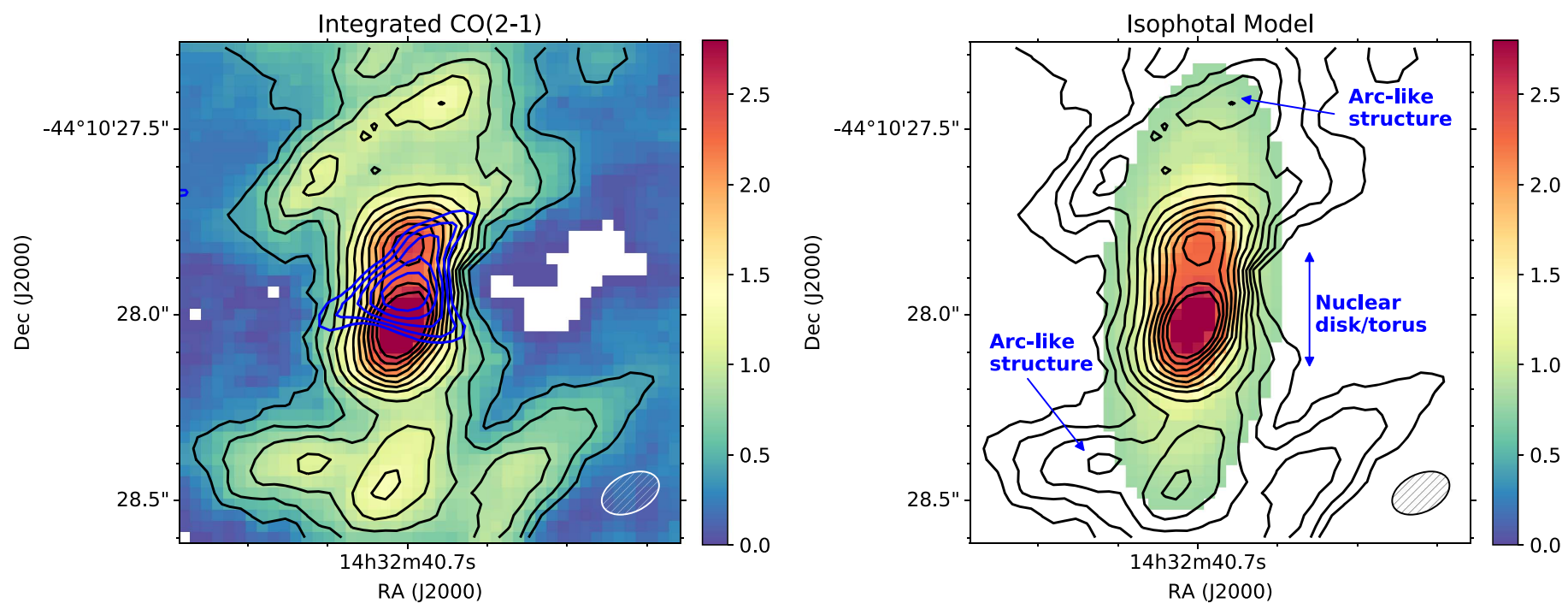

Figure 5. Left panel shows in color in a linear scale the observed ALMA CO(2-1) $b=-0.5$ integrated intensity map of the nuclear region of NGC 5643. The black contours are also the $\mathrm{CO}(2-1)$ intensity in a linear scale with the lowest contour at $0.6 \mathrm{Jy} \mathrm{km} \mathrm{s}^{-1}$ beam $^{-1}$ and contour steps of $0.2 \mathrm{Jy} \mathrm{km} \mathrm{s} \mathrm{k}^{-1}$ beam ${ }^{-1}$. The blue contours are the Band 6 rest-frame $232 \mathrm{GHz}$ continuum. Right panel shows in color the isophotal model fitted to the nuclear disk $\mathrm{CO}(2-1)$ emission. The black contours are as in the left panel. We mark the location of the nuclear arc-like structures and the disk/torus. For the latter the size of the arrow indicates the measured FWHM in the north-south direction not corrected for the ALMA beam (ellipse, 0 .! $16 \times 0$.! 11 at $\mathrm{PA}_{\text {beam }}=-67^{\circ}$ ).
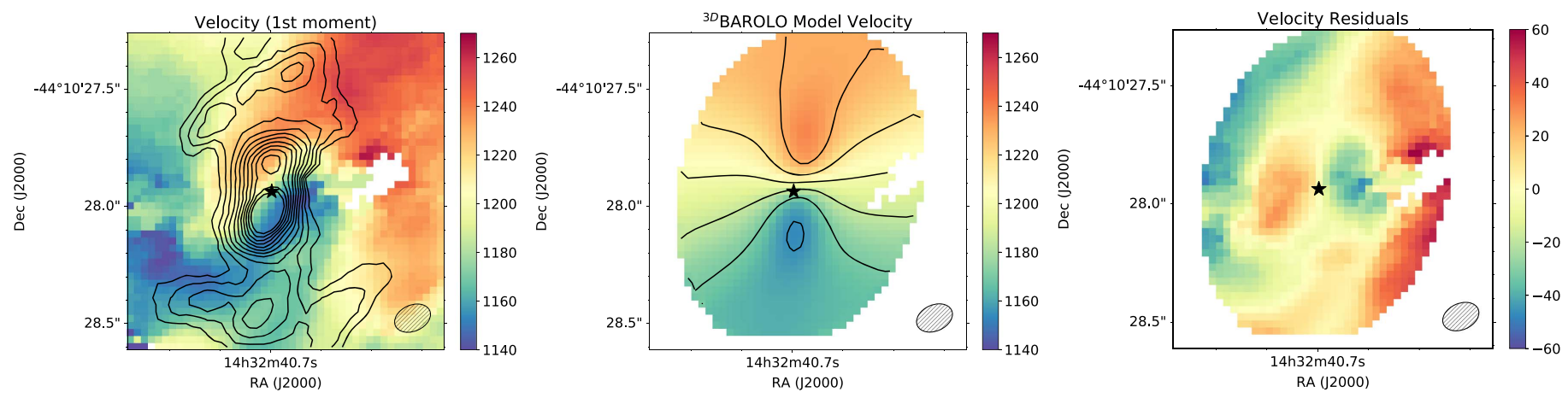

Figure 6. Left panel: ${ }^{3 \mathrm{D}} \mathrm{B}$ AROLO velocity field (1st moment map) from the $b=-0.5$ data cube. The black contours are $\mathrm{CO}(2-1)$ integrated line intensity. Middle panel: ${ }^{3 \mathrm{D}}$ BAROLO model of the velocity field without a radial velocity component. The contours are the isovelocities of the model. Right panel: residual velocity field resulting from subtracting the model from the observed velocity field. In all three panels, the FoV and angular resolution are as in Figure 5 and the star symbol marks the peak of the rest-frame $232 \mathrm{GHz}$ continuum. The vertical color bars indicate velocities in units of $\mathrm{km} \mathrm{s}^{-1}$.
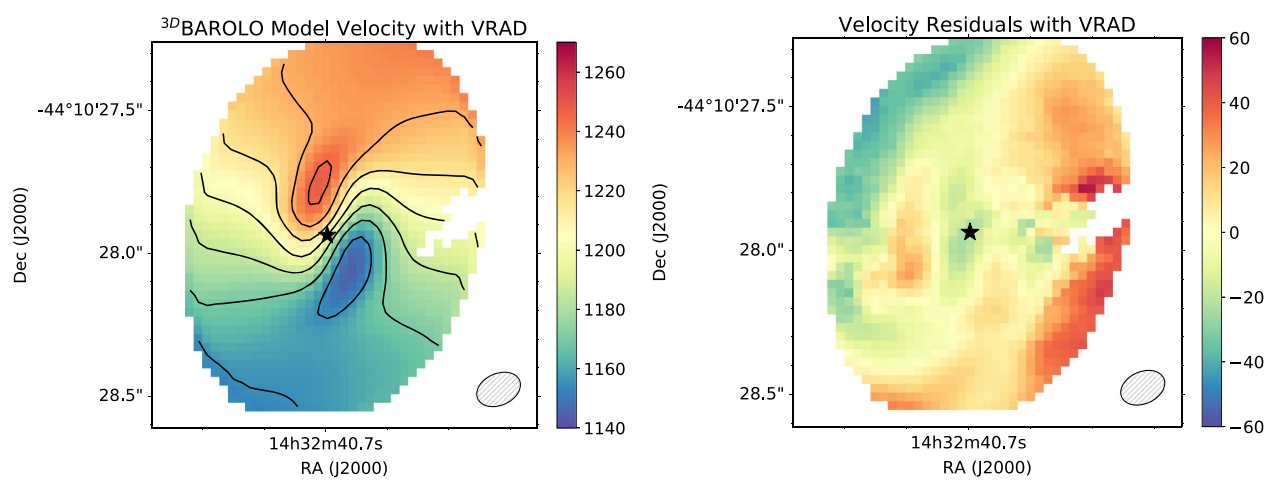

Figure 7. The two panels are the same as the middle and right panels of Figure 6, but the ${ }^{3 \mathrm{D}}$ BAROLO velocity field model includes a radial velocity component.

the order of $\pm 30 \mathrm{~km} \mathrm{~s}^{-1}$ or less. The typical axisymmetric value of the radial velocity fitted by ${ }^{3 \mathrm{D}} \mathrm{BAROLO}$ is of the order of $70 \mathrm{~km} \mathrm{~s}^{-1}$.

We also constructed position-velocity $(\mathrm{p}-\mathrm{v})$ diagrams taken along the kinematic major and minor axes of the nuclear $\mathrm{CO}(2-1)$ disk oriented, according to the fits obtained by ${ }^{3 \mathrm{D}} \mathrm{BAROLO}$, along $\mathrm{PA}^{\text {major }}=355^{\circ}$ and $\mathrm{PA}^{\text {minor }}=265^{\circ}$, respectively. We used an aperture size of 0.2 that is approximately equal to the beam size of the $b=-0.5$ data cube. As seen from Figure 8, the $\mathrm{CO}(2-1)$ emission in the nuclear disk is spread over a wide range of velocities more or less symmetrically around $v_{\text {sys }}=1198 \mathrm{~km} \mathrm{~s}^{-1}: \quad v-v_{\text {sys }} \simeq$ $[-160,+130] \mathrm{km} \mathrm{s}^{-1}$. The two $\mathrm{p}-\mathrm{v}$ diagrams illustrate the kinematic decoupling of the nuclear $\mathrm{CO}(2-1)$ disk relative to 

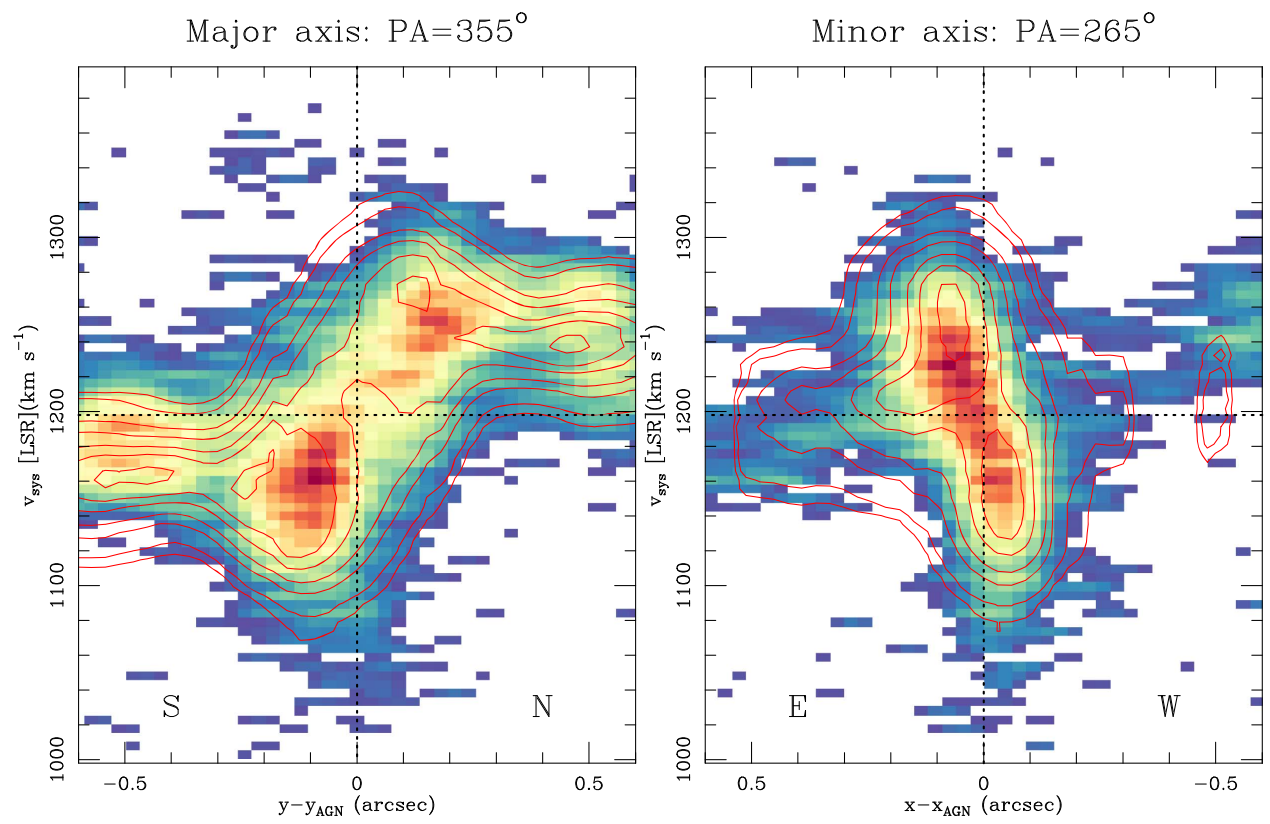

Figure 8. In color are the position-velocity diagrams of the nuclear region extracted with an aperture size of 0 !l $2^{2}$ along the kinematic major and minor axes determined with ${ }^{3 \mathrm{D}}$ BAROLO for the nuclear region (see Section 4.2). The horizontal lines are the systemic velocity fitted in the nuclear region and the vertical line represents the position of the AGN as traced by the $232 \mathrm{GHz}$ continuum peak. The red contours are the p-v diagrams derived from the ${ }^{3 \mathrm{D}} \mathrm{BAROLO}$ model with a radial velocity component (see Figure 7 and the text) at 1\%, 2.5\%, 10\%, 20\%, 40\%, 60\%, and 90\% of the peak value.

the larger-scale disk of the galaxy. The amplitude and even the sign of the characteristic mean line-of-sight velocities of the emission show a significant change beyond $r \simeq 0$ "! $3-0$ "! 4 $(30 \mathrm{pc})$ and $r \simeq 0$ !" $1-0$ !"2 (14 pc) in the major and minor-axis $\mathrm{p}-\mathrm{v}$ diagrams, respectively. These physical scales coincide with those derived from the morphology of the $\mathrm{CO}(2-1)$ intensity (Figure 5).

The behavior seen in the $\mathrm{p}-\mathrm{v}$ diagram identifies the transition from the nuclear disk to the larger-scale disk modeled in Section 3.2. Furthermore, the $\mathrm{CO}(2-1)$ nuclear disk shows strong non-circular motions. An inspection of the minor-axis $\mathrm{p}-\mathrm{v}$ diagram indicates that the maximum observed deviations from rotational motions reach $\pm 100 \mathrm{~km} \mathrm{~s}^{-1}$. The emission appears blueshifted (redshifted) on the western (eastern) side of the nuclear disk, as also seen in the mean-velocity residual map in Figure 6. In Figure 8, we also plotted as contours the p-v diagram resulting from the ${ }^{3 \mathrm{D}} \mathrm{BAROLO}$ fit with the radial velocity component. It is clear that this model is able to reproduce fairly well the non-circular motions in the nuclear region. We discuss the possible interpretations of this radial velocity component in Section 5.2.

\subsection{Molecular Gas Mass}

We measured a $\mathrm{CO}(2-1)$ line intensity over the nuclear region approximately of $20 \mathrm{Jy} \mathrm{km} \mathrm{s}^{-1}$ over a region of $\sim 0$ " $4 \times 0$." 6 . Assuming a $\mathrm{CO}(1-0) / \mathrm{CO}(2-1)$ brightness temperature ratio of one and using the relation of Sakamoto et al. (1999) with a Galactic CO-to- $\mathrm{H}_{2}$ conversion factor of $X=2 \times 10^{20} \mathrm{~cm}^{-2}\left(\mathrm{~K} \mathrm{~km} \mathrm{~s}^{-1}\right)^{-1}$ we derived a molecular gas mass in the nuclear disk of $M\left(\mathrm{H}_{2}\right)=$ $1.1 \times 10^{7} M_{\odot}$. The typical uncertainty for the X-factor is $2-3$ for gas clouds in our Galaxy (Bolatto et al. 2013). The nuclear disk of NGC 5643 is about a factor of 10 more massive than the torus in NGC 1068 (García-Burillo et al. 2016; Imanishi et al. 2018). This is not surprising given the different sizes measured in $\mathrm{CO}(2-1)$ for
NGC 5643 (see Section 4.1) and in NGC 1068 using dense molecular gas tracers (García-Burillo et al. 2016; Imanishi et al. 2018). However, the size of the torus of NGC 1068 measured in the $\mathrm{CO}(2-1)$ and $\mathrm{CO}(3-2)$ transitions is about $20 \mathrm{pc}$ and thus comparable to NGC 5643 (S. García-Burillo et al. 2018, in preparation). On the other hand, the derived molecular gas mass of the nuclear disk in NGC 5643 agrees well with the average gas mass for a sample of Seyfert galaxies from dynamical constraints assuming a gas fraction of $10 \%$ (Hicks et al. 2009).

We also estimated the dynamical mass enclosed in the nuclear disk regions by assuming a spheroid, $M_{\mathrm{dyn}}(R)=2.32 \times$ $10^{5} R V^{2}(R)$ in $M_{\odot}$ with $R$ expressed in kpc, $V$ in $\mathrm{km} \mathrm{s}^{-1}$ (Lequeux 1983). For the velocity we took the terminal value of $v_{\text {term }}=100 \mathrm{~km} \mathrm{~s}^{-1} / \sin (i)=115 \mathrm{~km} \mathrm{~s}^{-1}$ at $r=0.1-0.12$ (see Figure 8), which is more appropriate for inclined disks. This leads to a dynamical mass of approximately $5 \times 10^{7} M_{\odot}$, which would imply a gas fraction of approximately $20 \%$.

The high molecular gas surface density in the nuclear disk of NGC 5643 would imply some star formation activity in the nuclear region as found for other Seyferts (Hicks et al. 2009). The detection of emission from the $11.3 \mu \mathrm{m}$ polycyclic aromatic hydrocarbon feature in the central 0." 4-0!'7 33-57 pc (see Hönig et al. 2010; González-Martín et al. 2013) might be indicating ongoing or recent star formation activity in the nuclear region.

\subsection{Column Density}

Having the molecular gas mass in the disk and the approximate size we computed an average column density of $N\left(\mathrm{H}_{2}\right) \sim 4 \times$ $10^{23} \mathrm{~cm}^{-2}$. This value is the column density averaged over the nuclear disk size of $\sim 0$ ". $4 \times 0$ ". 6 . The averaged column density is similar to those derived for Seyfert galaxies by Hicks et al. (2009) on similar physical scales using the near-infrared $2.12 \mu$ $\mathrm{H}_{2}$ line. If we only focus on the AGN position, as given by the $232 \mathrm{GHz}$ continuum peak (see Figure 5) and at our angular 


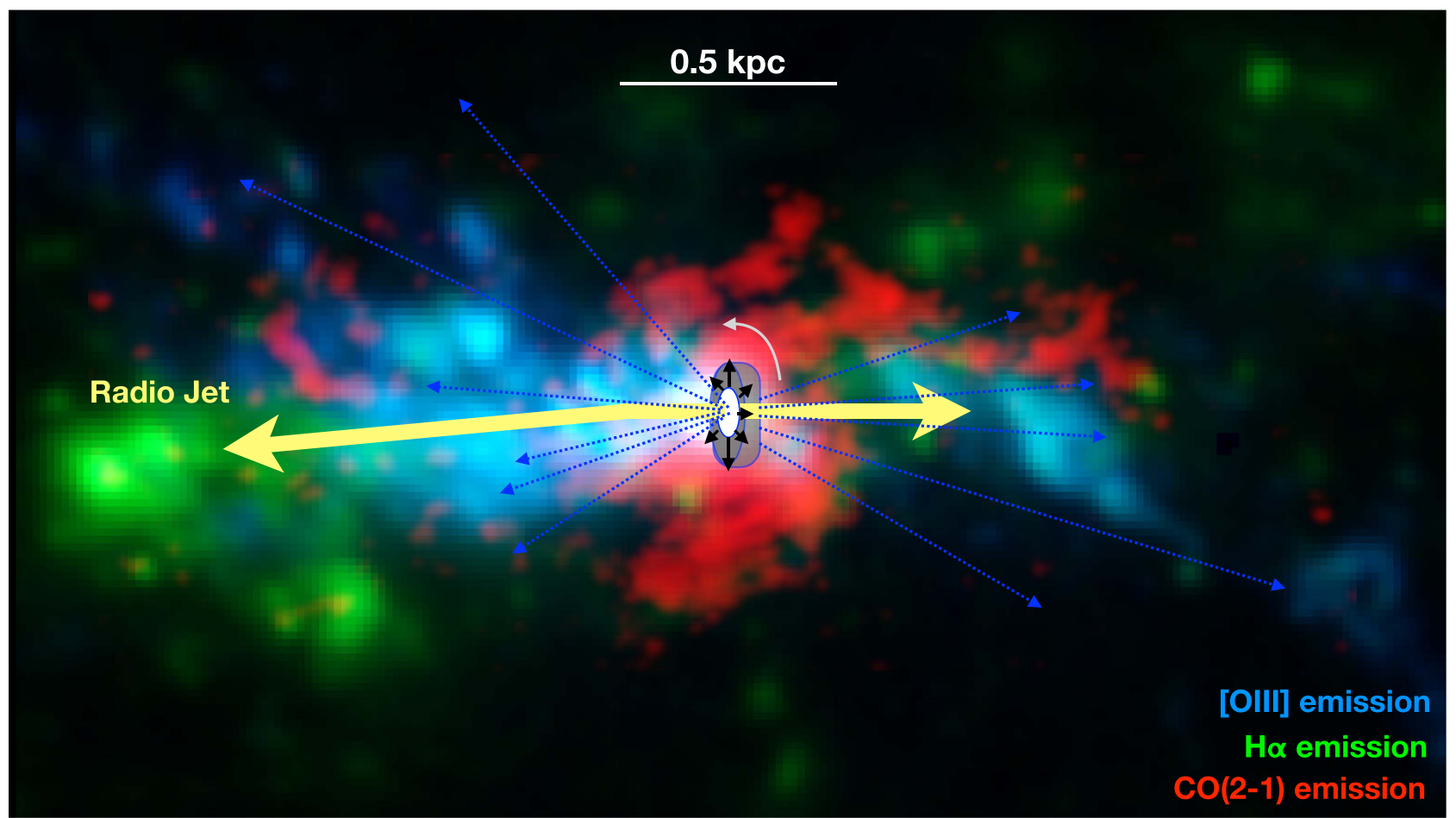

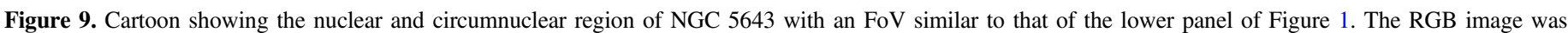

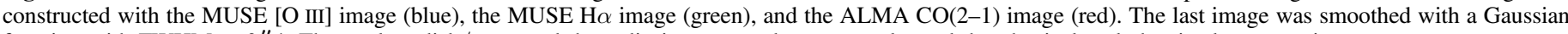
function with FWHM $\sim 0$." 4 . The nuclear disk/torus and the radio jet are not drawn to scale, and the physical scale bar is also approximate.

resolution, we would derive a column density of $N\left(\mathrm{H}_{2}\right) \sim 5 \times$ $10^{23} \mathrm{~cm}^{-2}$. At the peak of the $\mathrm{CO}(2-1)$ emission the value of the column density is $N\left(\mathrm{H}_{2}\right) \sim 7 \times 10^{23} \mathrm{~cm}^{-2}$.

The derived $\mathrm{H}_{2}$ column density is short of the value derived from X-ray observations that provides a lower limit for the obscuring column density toward the AGN in NGC 5643 of $N_{\mathrm{H}}(\mathrm{los})>5 \times 10^{24} \mathrm{~cm}^{-2}$ (Annuar et al. 2015). However, the X-ray column is modeled through a narrow pencil beam toward the accretion disk. For type 2 AGNs one would expect it to intersect high column clouds in the torus (or even the broad-line region). On the other hand, the column measured from our ALMA data is averaged over a larger area that is defined by the beam of $\sim 13 \mathrm{pc} \times 9 \mathrm{pc}$ of the observations.

Another source of uncertainty in this comparison is the assumed CO-to- $\mathrm{H}_{2}$ conversion factor. Wada et al. (2018) computed the $\mathrm{CO}$ line chemistry from their radiation-driven fountain model fitted to the Circinus galaxy to derive the CO-to- $\mathrm{H}_{2}$ conversion factor. They demonstrated that this conversion factor depends strongly on the integrated intensity of the line for a given line of sight. For the brightness temperature derived at the location of the AGN or the peak of the $\mathrm{CO}(2-1)$ emission of NGC 5643 the predictions for CO-to- $\mathrm{H}_{2}$ conversion factor for the $\mathrm{CO}(2-1)$ line are 5-10 higher than the Galactic value with a large scatter (see their Figure 5). We note that the inclinations of NGC 5643 and Circinus are similar $\left(i \sim 60^{\circ}-65^{\circ}\right.$ versus $\left.i \sim 75^{\circ}\right)$. We note, however, that empirically the $\mathrm{CO}$ conversion factor tends to be lower in the centers of galaxies (Sandstrom et al. 2013).

\section{Discussion and Conclusions}

\subsection{Circumnuclear Scales}

To further understand the connection between the cold molecular gas in the circumnuclear region of NGC 5643 and the outflow detected in ionized gas we produced a red-greenblue (RGB) image using the MUSE [O III] image in blue, the MUSE $\mathrm{H} \alpha$ image in green (see Section 2.2), and the ALMA $\mathrm{CO}(2-1)$ image in red. The result is shown in Figure 9 with an FoV approximately equal to that of the lower panel of Figure 1. The RGB image nicely shows the ionization cone as traced by the [O III] emission on the east side of the galaxy. Close to the AGN position the [O III] emission from the counter-cone to the west is obscured by material in the host galaxy that is traced by the $\mathrm{CO}(2-1)$ nuclear spiral. The $\mathrm{H} \alpha$ emission on this side of the galaxy is less obscured, as expected (see Cresci et al. 2015 for an in-depth discussion).

Fischer et al. (2013) interpreted the extended [O III] emission observed in some Seyfert galaxies as the result of the intersection between the disk of the host galaxy and the bicone. From their modeling of the NLR kinematics of NGC 5643 they inferred an angle between the NLR bicone axis and the normal to the host galaxy disk of $42^{\circ}$. We derived an angle difference between the normals of the galaxy disk and the nuclear disk fitted with ${ }^{3 \mathrm{D}} \mathrm{BAROLO}$ of $25^{\circ}\left(30^{\circ}\right.$ if we take the value they used for the inclination of the galaxy). They also derived a cone half outer opening angle of $\theta_{\max }=55^{\circ}$. This implies that this rather wide ionization cone would intercept the host galaxy but only to the southeast of the AGN where the radio jet is also impacting on the host galaxy and producing positive feedback (Cresci et al. 2015).

To the northeast of the galaxy nucleus, some of the [O III] filaments are coincident with the regions with $\mathrm{CO}(2-1)$ with redshifted velocity components (see Figures 2 and 4 ) and are mostly along the minor axis of the host galaxy. As interpreted for the hot molecular gas $\mathrm{H}_{2}$ emission by Davies et al. (2014), these components are probably due to gas excited on the edge of the cone. The spiral morphology of the $\mathrm{CO}(2-1)$ emission suggests that this emission is in the disk of the galaxy, and so is 
the gas with the redshifted velocity component. With this geometry the non-circular motions are due to molecular gas being pushed outward in the disk of the galaxy (note that northeast is the far side of the galaxy disk and southwest the near side) by the AGN wind near the fourth spiral arm in the circumnuclear region. The region with the blueshifted velocity component to the southwest of the AGN can also be explained as gas being pushed outward by the ionized outflow and moving toward us. A similar interaction between the outflow detected in ionized gas and the cold molecular gas in the galaxy disk with blueshifted and redshifted velocity components has been observed in the Circinus galaxy (Zschaechner et al. 2016).

We also plotted in the cartoon of Figure 9 the approximate orientation of the radio emission/jet (Morris et al. 1985; Leipski et al. 2006) pointing to the location of the $\mathrm{H}$ II regions with bright $\mathrm{H} \alpha$ emission. Cresci et al. (2015) proposed that these regions are the result of positive feedback induced by gas compression by the AGN outflow. Although faint, we detected $\mathrm{CO}(2-1)$ emission at the location of these star-forming clumps. The passage of the radio jet through the disk of the galaxy might be responsible for clearing the $\mathrm{CO}(2-1)$ molecular gas in the eastern side spiral arm in a region $\sim 5^{\prime \prime}$ the nucleus of NGC 5643 (see Figure 1).

\subsection{Nuclear Scales}

We have detected a massive $\left(M\left(\mathrm{H}_{2}\right)=1.1 \times 10^{7} M_{\odot}\right)$ nuclear disk in NGC 5643. The size of the nuclear disk measured from the Band $6 \mathrm{CO}(2-1)$ integrated emission is approximately $26 \mathrm{pc}$ (FWHM). This is in fairly good agreement with the typical sizes (radius of $30 \mathrm{pc}$ ) derived for nuclear disks from hot molecular gas $\mathrm{H}_{2} 2.12 \mu \mathrm{m}$ by Hicks et al. (2009). The disk/torus of NGC 5643 is a factor of two-to-three larger than that detected by ALMA in $\mathrm{CO}(6-5)$ in the Seyfert 2 galaxy NGC 1068 ( 7-10 pc; see García-Burillo et al. 2016). One possible explanation is that the $\mathrm{CO}(6-5)$ line traces denser gas than $\mathrm{CO}(2-1)$ and thus it probes molecular gas that needs to be closer to the AGN to be excited. Indeed, our new ALMA $\mathrm{CO}(2-1)$ and $\mathrm{CO}(3-2)$ observations of NGC 1068 reveal a larger structure (diameter $\sim 20 \mathrm{pc}$ ) than seen in $\mathrm{CO}(6-5)$ that is also well connected with the circumnuclear disk (S. GarcíaBurillo et al. 2018, in preparation). The molecular gas column density averaged over the ALMA beam of our observations and at the AGN location is $\sim 5 \times 10^{23} \mathrm{~cm}^{-2}$. The material in the nuclear disk is likely responsible, at least in part, for obscuring the AGN in NGC 5643.

The modeling of the $\mathrm{CO}(2-1)$ kinematics in the nuclear region clearly implies the presence of non-circular motions, with a maximum amplitude of $100 \mathrm{~km} \mathrm{~s}^{-1} / \sin (i)$, in the inner $r \sim 0$ !" 2 (16 pc). However, their interpretation requires a detailed knowledge of the nuclear potential and in particular whether there is a nuclear bar in NGC 5643 or not. Using nearinfrared imaging observations, Jungwiert et al. (1997) detected an isophotal twist between radial distances of $r=3^{\prime \prime}$ and $r=30^{\prime \prime}$. This feature could be indicative of the presence of a nuclear bar with $\mathrm{PA}=-49^{\circ}$. In fact, it is possible that the nuclear arc structures could be associated with such a bar, although they are on smaller scales (see Figure 5). In this scenario, the radial motions seen in the inner $1^{\prime \prime}$ could be interpreted as gas elliptical stream lines in a nuclear bar. In a dynamically decoupled nuclear bar, the observed kinematics could be explained as both inflowing and outflowing radial motions.
In the absence of a putatively decoupled nuclear stellar bar, the canonical gas response to the large-scale bar in the framework of the epicyclic approximation inside the inner Lindblad resonance would favor the presence of inward radial motions (see Figure 3 of Wong et al. 2004). Therefore, in a coplanar geometry with the nuclear disk (see the assumed orientation of the nuclear disk in Figure 9) the non-circular motions are not due to the large-scale bar and could be explained as due to material outflowing in the plane of the nuclear disk. This reproduces the blueshifted velocities on the western side of the nuclear disk and redshifted velocities on the eastern side of the nuclear disk (see $\mathrm{p}-\mathrm{v}$ along the minor axis of the nuclear disk in Figure 8). The ionized gas in NGC 5643 is outflowing (blueshifted emission at the AGN location in the MUSE [O III] emission) perpendicular to the nuclear disk (Cresci et al. 2015). The different location for the molecular gas and ionized gas is predicted by the radiationdriven fountain model simulations done for the Circinus galaxy (Wada et al. 2016, 2018). In particular, this model shows that most of the nuclear molecular gas emission is expected in the equatorial plane of the nuclear disk with no dense molecular winds outflowing along the rotational axis, while the ionized gas is outflowing perpendicular to the disk. A similar equatorial outflow has been observed in the Seyfert 2 galaxy NGC 5929 by Riffel et al. (2014) on scales even larger ( $300 \mathrm{pc})$ than in NGC 5643.

We conclude that the Band $6 \mathrm{CO}(2-1)$ observations have resolved a massive nuclear molecular gas rotating disk/torus in NGC 5643 with strong non-circular motions. The inclination of the nuclear disk agrees with the inclination required to model the ionization cone emission traced by [O III] and $\mathrm{H} \alpha$ emission (Fischer et al. 2013). Thus, the $\mathrm{CO}(2-1)$ disk/torus is likely collimating the ionization cone of NGC 5643 as well as obscuring the AGN. In the framework of the AGN Unified Model (Antonucci 1993), the detected disk could be interpreted as the obscuring torus.

We thank Enrico di Teodoro for providing advice to run ${ }^{3 \mathrm{D}} \mathrm{BAROLO}$ and an anonymous referee for suggestions that helped improve the paper.

A.A.-H. acknowledges support from the Spanish Ministry of Economy and Competitiveness through grant AYA201564346-C2-1-P, which was party funded by the FEDER program and from CSIC grant PIE201650E36. A.A.-H., M. P.-S., and A.B. acknowledge support from the Royal Society International Exchange Scheme under project IE160174. A.L. acknowledges support from the Spanish Ministry of Economy and Competitiveness through grant ESP2015-68964. M.P.-S. and D.R. acknowledge support from STFC through grant ST/N000919/1. T.D.-S. acknowledges support from ALMACONICYT project 31130005 and FONDECYT regular project 1151239. P.G. acknowledges support from STFC (ST/ J003697/2). A.H.-C. acknowledges funding by the Spanish Ministry of Economy and Competitiveness under grant AYA2015-63650-P. S.F.H. acknowledges support from the EU/Horizon 2020 ERC Starting Grant DUST-IN-THE-WIND (ERC-2015-StG-677117). C.R.A. acknowledges the Ramón y Cajal Program of the Spanish Ministry of Economy and Competitiveness through project RYC-2014-15779 and AYA 2016-76682-C3-2-P. D.R. acknowledges the support of the Science and Technology Facilities Council (STFC) through grant ST/P000541/1. 
This paper makes use of the following ALMA data: ADS/JAO.ALMA\#2016.1.00254.S. ALMA is a partnership of ESO (representing its member states), NSF (USA) and NINS (Japan), together with NRC (Canada), MOST and ASIAA (Taiwan), and KASI (Republic of Korea), in cooperation with the Republic of Chile. The Joint ALMA Observatory is operated by ESO, AUI/NRAO and NAOJ. Based on observations collected at the European Organisation for Astronomical Research in the Southern Hemisphere under ESO programme 095.B-0532(A).

Facilities: ALMA, ESO (VLT/MUSE).

\section{ORCID iDs}

A. Alonso-Herrero (iD https://orcid.org/0000-0001-6794-2519 M. Pereira-Santaella (iD https://orcid.org/0000-00024005-9619

S. García-Burillo (iD https://orcid.org/0000-0003-0444-6897

F. Combes (iD https://orcid.org/0000-0003-2658-7893

D. Asmus (D) https://orcid.org/0000-0003-0220-2063

T. Díaz-Santos (1) https://orcid.org/0000-0003-0699-6083

P. Gandhi (i) https://orcid.org/0000-0003-3105-2615

O. González-Martín (iD https://orcid.org/0000-00022356-8358

A. Hernán-Caballero (iD https://orcid.org/0000-00024237-5500

C. Ramos Almeida (iD https://orcid.org/0000-0001-8353-649X

C. Ricci (i) https://orcid.org/0000-0001-5231-2645

D. Rosario (ib https://orcid.org/0000-0002-0001-3587

\section{References}

Alonso-Herrero, A., Ramos Almeida, C., Mason, R., et al. 2011, ApJ, 736, 82 Annuar, A., Gandhi, P., Alexander, D. M., et al. 2015, ApJ, 815, 36 Antonucci, R. 1993, ARA\&A, 31, 473

Bacon, R., Accardo, M., Adjali, L., et al. 2010, Proc. SPIE, 7735, 773508

Baumgartner, W. H., Tueller, J., Markwardt, C. B., et al. 2013, ApJS, 207, 19

Bianchi, S., Guainazzi, M., \& Chiargerge, M. 2006, A\&A, 448, 499

Bolatto, A. D., Wolfire, M., \& Leroy, A. K. 2013, ARA\&A, 51, 207

Briggs, D. S. 1995, PhD thesis, New Mexico Institute of Mining and Technology

Burtscher, L., Meisenheimer, K., Tristram, K. R. W., et al. 2013, A\&A, 558, A149

Combes, F., García-Burillo, S., Casasola, V., et al. 2013, A\&A, 558, A124

Cresci, G., Marconi, A., Zibetti, S., et al. 2015, A\&A, 582, A63

Dale, D. A., Sheth, K., Helou, G., et al. 2005, AJ, 129, 2197

Davies, R. I., Maciejewski, W., Hicks, E. K. S., et al. 2014, ApJ, 792, 101

Davies, R. I., Mueller Sánchez, F., Genzel, R., et al. 2007, ApJ, 671, 1388

Di Teodoro, E. M., \& Fraternali, F. 2015, MNRAS, 451, 3921

Emsellem, E., Greusard, D., Combes, F., et al. 2001, A\&A, 368, 52

Esquej, P., Alonso-Herrero, A., González-Martín, O., et al. 2014, ApJ, 780, 86

Fischer, T. C., Crenshaw, D. M., Kraemer, S. B., \& Schmitt, H. R. 2013, ApJS, 209, 1

Fuller, L., Lopez-Rodriguez, E., Packham, C., et al. 2016, MNRAS, 462, 2618 Gallimore, J. F., Elitzur, M., Maiolino, R., et al. 2016, ApJL, 829, L7

García-Burillo, S., Combes, F., Schinnerer, E., Boone, F., \& Hunt, L. K. 2005 , A\&A, 441, 1011
García-Burillo, S., Combes, F., Ramos Almeida, C., et al. 2016, ApJL, 823, L12

García-Burillo, S., Combes, F., Usero, A., et al. 2014, A\&A, 567, A125

Gómez-Guijarro, C., González-Martín, O., Ramos Almeida, C., et al. 2017, MNRAS, 469, 2720

González-Martín, O., Rodríguez-Espinosa, J. M., Díaz-Santos, T., et al. 2013, A\&A, 553, A35

Greenhill, L. J., Kondratko, P. T., Lovell, J. E. J., et al. 2003, ApJL, 582, L11

Guainazzi, M., Rodriguez-Pascual, P., Fabian, A. C., Iwasawa, K., \& Matt, G. 2004, MNRAS, 355, 297

Hicks, E. K. S., Davies, R. I., Maciejewski, W., et al. 2013, ApJ, 768, 107

Hicks, E. K. S., Davies, R. I., Malkan, M. A., et al. 2009, ApJ, 696, 448

Ho, L. C., Li, Z.-H., Barth, A. J., Seigar, M. S., \& Peng, C. Y. 2011, ApJS, 197,21

Hönig, S. F., Kishimoto, M., Gandhi, P., et al. 2010, A\&A, 515, A23

Hopkins, P. F., \& Quataert, E. 2010, MNRAS, 407, 1529

Ichikawa, K., Packham, C., Ramos Almeida, C., et al. 2015, ApJ, 803, 57

Imanishi, M., Nakanishi, K., Izumin, T., \& Wada, K. 2018, ApJL, 853, L24

Izumi, T., Kawakatu, N., \& Kohno, K. 2016, ApJ, 827, 81

Jungwiert, B., Combes, F., \& Axon, D. J. 1997, A\&AS, 125, 479

Krause, M., Fendt, C., \& Neininger, N. 2007, A\&A, 467, 1037

Krips, M., Neri, R., García-Burillo, S., et al. 2007, A\&A, 468, L63

Leipski, C., Falcke, H., Bennert, N., \& Hüttemeister, S. 2006, A\&A, 455, 161

Lequeux, J. 1983, A\&A, 125, 394

Lin, M.-Y., Davies, R. I., Burtscher, L., et al. 2016, MNRAS, 458, 1375

López-Gonzaga, N., Jaffe, W., Burtscher, L., Tristram, K. R. W., \& Meisenheimer, K. 2014, A\&A, 565, A71

Maciejewski, W. 2004, MNRAS, 354, 892

Martini, P., Regan, M. W., Mulchaey, J. S., \& Pogge, R. W. 2003, ApJ, 589, 774

Martín-Vidal, I., Vlemmings, W. H. T., Muller, S., \& Casey, S. 2014, A\&A, 563, A136

McMullin, J. P., Waters, B., Schiebel, D., Young, W., \& Golap, K. 2007, in ASP Conf. Ser. 376, Astronomical Data Analysis Software and Systems XVI, ed. R. A. Shaw, F. Hill, \& D. J. Bell (San Francisco, CA: ASP), 127

Monje, R. R., Blain, A. W., \& Phillips, T. G. 2011, ApJS, 195, 23

Morris, S., Ward, M., Whittle, M., Wilson, A. S., \& Taylor, K. 1985, MNRAS, 216, 193

Mouri, H. 1994, ApJ, 427, 777

Mulchaey, J. S., Regan, M. W., \& Kundu, A. 1997, ApJS, 110, 299

Querejeta, M., Schinnerer, E., García-Burillo, S., et al. 2016, A\&A, 593, 118 Quillen, A. C., Alonso-Herrero, A., Rieke, M. J., et al. 1999, ApJ, 527, 696

Ramos Almeida, C., Levenson, N. A., Alonso-Herrero, A., et al. 2011, ApJ, 731, 92

Ramos Almeida, C., Martínez González, M. J., Asensio Ramos, A., et al. 2016, MNRAS, 461, 1387

Regan, M. W., \& Mulchaey, J. S. 1999, AJ, 117, 2676

Ricci, C., Ueda, Y., Koss, M. J., et al. 2015, ApJL, 815, L13

Riffel, R. A., Storchi-Bergmann, T., \& Riffel, R. 2014, ApJL, 780, L24

Sakamoto, K., Okumura, S. K., Ishizuki, S., \& Scoville, N. Z. 1999, ApJS, 124,403

Salak, D., Tomiyasu, Y., Nakai, N., Kuno, N., et al. 2017, ApJ, 849, 90

Sandstrom, K. M., Leroy, A. K., Walter, F., et al. 2013, ApJ, 777, 5

Sani, E., Davies, R. I., Sternberg, A., et al. 2012, MNRAS, 424, 1963

Schinnerer, E., Eckart, A., \& Tacconi, L. J. 2000, ApJ, 533, 826

Simpson, C., Wilson, A. S., Bower, G., et al. 1997, ApJ, 474, 121

Tacconi, L. J., Genzel, R., Blietz, M., et al. 1994, ApJL, 426, L77

Tristram, K. R. W., Raban, D., Meisenheimer, K., et al. 2009, A\&A, 502, 67

Wada, K., Fukushige, R., Izumi, T., \& Tomisaka, K. 2018, ApJ, 852, 88

Wada, K., Schartmann, M., \& Meijerink, R. 2016, ApJL, 828, L19

Wong, T., Blitz, L., \& Bosma, A. 2004, ApJ, 605, 183

Zschaechner, L. K., Walter, F., Bolatto, A., et al. 2016, ApJ, 832, 142 